PREHOSPITAL CARE

\title{
Levels of mental health problems among UK emergency ambulance workers
}

\author{
P Bennett, Y Williams, N Page, K Hood, M Woollard
}

Emerg Med J 2004;21:235-236. doi: 10.1136/emj.2003.005645

This study examined the prevalence of post-traumatic stress disorder (PTSD), depression, and anxiety in a sample of emergency ambulance personnel. Of 1029 personnel in one ambulance service mailed a questionnaire, 617 were returned. Among respondents, the overall rate of PTSD was $22 \%$. Levels of PTSD did not differ according to grade, but men had a higher prevalence rate than women $123 \%$ compared with 15\%). Nearly one in ten reported probable clinical levels of depression, $22 \%$ reported probable clinical levels of anxiety based on Hospital Anxiety and Depression Scale scores.

$\mathrm{D}$ espite being identified as a "high risk" occupational group, there is a lack of data concerning the prevalence of emotional problems among emergency ambulance personnel. Previous studies have identified a prevalence of post-traumatic stress disorder (PTSD) of between $20 \%$ and $21 \%,{ }^{12}$ and one third of ambulance personnel to evidence some degree of psychiatric morbidity. ${ }^{3}$ Clohessy and Ehlers, ${ }^{1}$ for example, found that $22 \%$ of their sample of 56 ambulance workers met the General Health Questionnaire screening criteria for psychiatric symptoms. Thirty two per cent of Alexander and Klein's ${ }^{3}$ sample of 110 Scottish ambulance workers achieved the same criterion. These data must be considered preliminary, however, as they are based on comparatively small, ${ }^{1-3}$ volunteer ${ }^{12}$ samples, with unclear sampling frames, ${ }^{2}$ or have reported non-specific psychopathology. ${ }^{3}$ There is, therefore, a need to identify the prevalence of PTSD and other emotional disorders among emergency personnel. This paper reports prevalence data from the first large scale population study of the prevalence of PTSD, depression, and anxiety in this population.

\section{PARTICIPANTS, METHOD, RESULTS}

The sample comprised all emergency medical technicians (EMTs) and paramedics working for one ambulance service serving a population of about three million people in a combination of rural and urban settings. To maintain anonymity, no central register matching names to questionnaire numbers was kept. Accordingly, two questionnaires were sent to all 1029 potential participants: an initial questionnaire and reminder sent three weeks later. A total of 617 questionnaires were returned: a response rate of $60 \%$. The final sample comprised 194 EMTs and 380 paramedics. Forty three respondents did not report their grade. There were 513 male and 91 female respondents. Thirteen did not report their sex. The male/female ratio is similar to that found throughout the service, suggesting there was no sex bias in responding to the questionnaire. The mean age of respondents was 39.58 years $(S D=10.60)$.
The questionnaire included two single yes/no items measuring the presence of intrusive work related memories either in the present or past. Participants with such memories for at least one month, completed the Posttraumatic Diagnostic Scale (PDS) ${ }^{4}$ which is validated against psychiatric ratings and achieves an $82 \%$ concordance with psychiatric interview. Scores indicate severity, while the pattern of symptoms reported contributes to the diagnosis of PTSD. Thirty two women and 261 men completed the PDS. All participants completed the Hospital Anxiety and Depression Scale (HADS), ${ }^{5}$ which has 14 items each with a four point severity scale, and provides cut off scores of 11 or more that indicate "probable" diagnoses of clinical anxiety and depression.

Key findings were that about two thirds of the sample reported experiencing intrusive and troubling work related thoughts either now or in the past. There were no differences in the reporting of present troubling memories according to grade (see table 1), although a higher percentage of paramedics than EMTs reported having had them in the past $\left(\chi^{2}=3.175, \mathrm{p}<0.05\right)$. Twenty two per cent $(95 \%$ CI 19 to 26) of the sample had PDS scores indicative of a diagnosis of PTSD. No difference in the prevalence of PTSD between EMTs and paramedics was found. However, men had a higher prevalence rate than women $\left(\chi^{2}=4.67, \mathrm{p}<0.05\right)$. Nearly $10 \%$ of participants reported probable clinical levels of depression, $22 \%$ reported probable clinical levels of anxiety based on HADS scores. There were no sex differences in levels of reported anxiety or depression.

\section{COMMENT}

These data compare with those from other studies in smaller or unrepresentative studies, ${ }^{1-3}$ suggesting a stable prevalence rate for PTSD across services of just over $20 \%$, a $10 \%$ level of depression and $22 \%$ level of anxiety. Some caveats should be given to these findings. Firstly, they are self report and based on questionnaires. Secondly, while the response rate of $60 \%$ is good for this type of survey, it still allows the possibility of response bias. What biases may have affected the reported prevalence levels reported is unclear. It is possible that people who experienced high levels of PTSD avoided completing the questionnaire: a characteristic central to the condition. It is also possible that all those not returned were sent to people not experiencing any problems, and who therefore saw no value in completing the questionnaire. To investigate any trends in the data that may have provided an indicator of which of these biases were most dominant, the number of PTSD cases identified in each consecutive group of 20 questionnaires returned time was examined. This showed a

Abbreviations: PTSD, post-traumatic stress disorder; EMT, emergency medical technician; PDS, Posttraumatic Diagnostic Scale; HADS Hospital Anxiety and Depression Scale 


\begin{tabular}{|c|c|c|c|c|}
\hline & Paramedic & EMT & Male & Female \\
\hline \multicolumn{5}{|l|}{ Troubling memories } \\
\hline Now & 45.8 & 43.9 & 37.7 & 46.3 \\
\hline Past & 62.4 & $55.8^{*}$ & 61 & 60.8 \\
\hline \multicolumn{5}{|l|}{ PTSD } \\
\hline Mean (SD) & $17.75(11.65)$ & $20.46(11.96)$ & 18.18 (12.93) & $18.67(11.7)$ \\
\hline Diagnostic criteria & 21.8 & 22.2 & 15.2 & $23.1^{*}$ \\
\hline \multicolumn{5}{|l|}{ Anxiety } \\
\hline Mean (SD) & $7.9(4.11)$ & $8.6(4.62)$ & $8.10(4.51)$ & $8.13(4.3)$ \\
\hline Clinically significant & 23 & 24 & 19 & 24.7 \\
\hline \multicolumn{5}{|l|}{ Depression } \\
\hline Mean (SD) & $5.2(3.83)$ & $5.6(4.72)$ & $4.99(4.62)$ & $5.37(4.10)$ \\
\hline Clinically significant & 8 & 10 & 11.3 & 8.2 \\
\hline
\end{tabular}

uniform prevalence rate throughout the response period, indicating no obvious bias to over-report or under-report PTSD levels.

Among the women who took part in the survey, prevalence rates for PTSD were lower than for men, while rates of depression and anxiety did not differ significantly. Although the comparatively small number of women in the sample means that these findings should be considered with some caution, these data are of interest, particularly as they are against the trends found in the general population. ${ }^{6}$ The reasons for our findings are not clear. It is possible that women who are particularly hardy self select into the ambulance service or that female ambulance personnel had better access to, or use of, social support or other coping mechanisms than the men. These hypotheses require further exploration.

A key imperative for any emergency service is to develop strategies for both the prevention and treatment of the significant levels of mental health problems associated with emergency work. Such interventions need to take into account both the severity and magnitude of the problem. This may require considerable innovation, particularly as the efficacy of some preventive methods has been significantly challenged. In the case of PTSD, for example, there are consistent findings that critical incident debriefing, a widely used preventive treatment, seems to compound rather than ameliorate the condition. ${ }^{7}$ A number of treatment and prevention approaches may therefore be need to be identified for each of the identified disorders. In addition, as wider organisational factors may also be implicated in the aetiology of PTSD and mood disorders in this population, ${ }^{1}$ consideration should be given to how organisational factors contribute to levels of emotional distress, and be included in any preventive strategy. Whatever approaches are used, they need to be simple and practical enough to be applicable to large numbers of workers.

\section{Authors' affiliations}

P Bennett, Bristol Doctoral Clinical Psychology Training Programme, Universities of Plymouth and Exeter, UK

Y Willia, Welsh Ambulance Trust, UK

N Page, Department of Psychology, Cardiff University, UK

K Hood, Department of General Practice, University of Wales College of Medicine, UK

M Woollard, Pre-Hospital Emergency Research Unit, University of Wales College of Medicine

Correspondence to: Dr P Bennett, Bristol Doctoral Clinical Psychology Training Programme, University of the West of England, Canon Kitson Building, St Matthias Campus, Bristol BS16 2JP, UK; Paul2 Bennett@ uwe.ac.uk

Accepted for publication 29 April 2003

\section{REFERENCES}

1 Clohessy S, Ehlers A. PTSD symptoms, responses to intrusive memories and coping in ambulance service workers. Br J Clin Psychol 1999;38:251-65.

2 Grevin F. Posttraumatic stress disorder, ego defense mechanisms, and empathy among urban paramedics. Psychol Rep 1996;79:483-95.

3 Alexander DA, Klein S. Ambulance personnel and critical incidents. Impact of accident and emergency work on mental health and emotional well-being. Br J Psychiatry 2001;178:78-81.

4 Foa EB, Cashman L, Jaycox L, et al. The validation of a self-report measure of posttraumatic stress disorder: the Posttraumatic Diagnostic Scale. Psychol Assess 1997:9:445-51.

5 Zigmond AS, Snaith RP. The Hospital Anxiety and Depression Scale. Acta Psychiatr Scand 1983;67:361-70.

6 Keller MB, Klerman GL, Lavori PW, et al. Long-term outcome of episodes of major depression: clinical and public health significance. JAMA 1984;252:788-92

7 Wessely S, Rose S, Bisson J. Brief psychological interventions ("debriefing") for trauma-related symptoms and the prevention of post traumatic stress disorder. Cochrane Library. Issue 3. Oxford: Update Software, 2000. 